\title{
DETERMINAÇÃO DE VALORES NORMAIS DA PRESSÃO INTRA-ABDOMINAL DE CÃES PELA CATETERIZAÇÃO VESICAL COM SONDA URETRAL DE P.V.C
}

\author{
(Determination of intra-abdominal normal pressure values in dogs using P.V.C urethral \\ catheter)
}

Rebeca Bacchi-Villanova, Eros Luiz de Souza, Tatiana Giordano Nerone, Bruna Berardi Guimarães, Mantheus Barbosa Gomes Cruz, Ricardo Guilherme D’Otaviano de Castro Vilani $^{1}$

${ }^{1}$ Correspondência: vilani@ufpr.br

RESUMO: A pressão intra-abdominal define-se como um estado de pressão na cavidade abdominal, o qual é determinado pelo índice de massa corporal, pela postura, atividade muscular da parede abdominal e pela respiração. O método padrão ouro para mensuração da pressão intra-abdominal recomendado pela Sociedade Mundial de Síndrome do Compartimento Abdominal é o método indireto por meio da sondagem vesical com sonda de Foley e infusão de $25 \mathrm{~mL}$ de solução fisiológica na bexiga. O desenvolvimento de uma técnica de aferição da PIA por meio de sonda uretral de policloreto de vinila (P.V.C.), torna essa técnica mais prática e fácil de ser realizada em Medicina Veterinária. Objetivou-se neste estudo, realizar a mensuração da PIA em 29 cães anestesiados saudáveis, com uma técnica que utiliza a sonda uretral de P.V.C. e a infusão de $1 \mathrm{~mL} / \mathrm{Kg}$ de solução fisiológica, correlacionando os valores encontrados com o peso dos animais. A média da PIA de cadelas anestesiadas com propofol e isoflurano pelo método proposto foi de $1,78 \mathrm{cmH} 20( \pm 1,39)$ e a mediana de $1,9 \mathrm{cmH} 20$. Os valores da PIA tem relação direta com o peso dos animais.

Palavras-chave: canino; hipertensão intra-abdominal; método vesical; sonda de Foley

ABSTRACT: The intra-abdominal pressure is defined as a state of pressure in the abdominal cavity, which is determined by the body mass index, the posture, muscular activity of the abdominal wall and breathing. The gold standard method for measurement of the intra-abdominal pressure recommended by the World Society of the Abdominal Compartment Syndrome is the indirect method by vesical catheterization with Foley catheter and infusion of $25 \mathrm{~mL}$ saline solution in the bladder. The development of a technique to measure intra-abdominal pressure by P.V.C. urethral catheter turn this technique into a more practical and easier one to be performed in Veterinary Medicine. The objective of this study was to measure the intra-abdominal pressure in 29 healthy anesthetized dogs, with a technique that uses the P.V.C. urethral catheter and the infusion of $1 \mathrm{~mL} / \mathrm{Kg}$ saline solution, correlating the values found with the weight of the animals. The average of the intraabdominal pressure of anesthetized dogs with propofol and isoflurane by the proposed method was $1,78 \mathrm{cmH} 20( \pm 1,39)$ and median of $1,9 \mathrm{cmH} 20$. The values of intra-abdominal pressure are directly related to the weight of the animals.

Key Words: canine; intra-abdominal hypertension; Foley urethral cateter; vesical method 


\section{INTRODUÇÃO}

A pressão intra-abdominal (PIA) define-se como um estado de pressão na cavidade abdominal, o qual é determinado pelo índice de massa corporal, pela postura, atividade muscular da parede abdominal e pela respiração (Castelhanos, Piñero e Fernández, 2007). O aumento de volume em qualquer um dos seus conteúdos irá levar a alterações da PIA, prejudicando a circulação sanguínea e, dessa forma, alterando a função e ameaçando a vitalidade dos tecidos (Chen et al., 2008).

Atualmente, sabe-se que valores de PIA acima de $12 \mathrm{mmHg}$ em pessoas são considerados como hipertensão intra-abdominal (HIA) e o aumento sustentado desta pressão a partir de 20 $\mathrm{mmHg}$ associado a uma disfunção orgânica, caracteriza síndrome do compartimento abdominal, que pode ser fatal (Malbrain et al., 2006).

Qualquer afecção abdominal traumática ou não é capaz de causar alterações na PIA. Além disso, a fluidoterapia agressiva é capaz de causar edema dos órgãos abdominais e culminar com aumento desta pressão (Cohen et al., 2003; Prado et al., 2005; Ball et al., 2008). Em cães e gatos pouco há descrito sobre fatores potencialmente capazes de aumentar a PIA, porém acredita-se que os mesmos fatores etiológicos relatados na medicina podem ser adotados na medicina veterinária (Drellich, 2000; Fetner e Prittie, 2012).

Diante disso, é fundamental o diagnóstico precoce da HIA/SCA, o qual é simples e baseia-se nos achados clínicos compatíveis com abdome tenso e distendido, oligúria progressiva e hipóxia. Estes sinais, porém, são considerados pouco sensíveis (Otto et al., 2008).

O método indireto de aferição da PIA por meio da medida da pressão vesical confirma o diagnóstico. Os métodos indiretos para medir a PIA, já foram realizados em quase todos os espaços dentro da cavidade abdominal: bexiga, estômago, cavidade uterina, reto e cateter colocado na veia cava inferior (Malbrain e Jones, 2006). No entanto, o método indireto mais utilizado desde a década de 80 e, a partir de 2006, consagrado como padrão ouro pela Sociedade Mundial de Síndrome do Compartimento Abdominal é o método vesical através da cateterização uretral com sonda de Foley a partir da instilação de $25 \mathrm{~mL}$ de solução fisiológica no interior da bexiga (Malbrain et al., 2006).

A bexiga é um reservatório passivo, que quando apresenta um volume pequeno, não é capaz de deflagar contrações das fibras do músculo detrusor, refletindo as pressões do interior do abdômen, a partir de um catéter intravesical (Andrade, 1998). As mensurações indiretas são baseadas na suposição de que a pressão hidrostática abdominal é uniformemente distribuída ao longo de todos os fluidos presentes no interior dos espaços da cavidade abdominal (Onichimowski, 2010).

Poucos trabalhos realizaram análise epidemiológica dos valores normais de PIA para cães. Em todos os estudos realizados nesta espécie, foi utilizada a sonda de Foley para sondagem vesical (Conzemius et al., 1995; Lopes, 2010; Way e Monnet, 2014). O desenvolvimento de uma técnica de aferição da PIA por meio de sonda uretral de P.V.C, torna essa técnica mais prática e fácil de ser realizada em Medicina Veterinária.

Objetivou-se neste estudo, realizar a mensuração da PIA em cães anestesiados saudáveis, desenvolvendo uma técnica que utiliza a sonda uretral de P.V.C e correlacionar os valores encontrados com o peso dos animais.

MATERIAL E MÉTODOS 
O estudo experimental prospectivo foi realizado em 29 cadelas encaminhadas para ovariohisterectomia eletiva na Unidade de Ensino em Saúde Animal I da Faculdade Evangélica do Paraná. Foi aprovado pelo Comitê de Ética em Pesquisa da Sociedade Evangélica Beneficente de Curitiba sob o número de protocolo 7586/10 em 14 de setembro de 2010.

As cadelas possuíam peso variando entre 13 e $37,4 \mathrm{~kg}$, de diferentes padrões raciais e foram consideradas saudáveis após avaliação clínica e nenhuma alteraçã o com relação a valores considerados normais para a espécie nos seguintes exames complementares: hemograma, contagem de plaquetas, pesquisa de hemoparasitas e dosagens bioquímicas de alanina aminotransferase (ALT), fosfatase alcalina (FA), uréia, creatinina e albumina.

As cadelas foram pré-medicadas com acepromazina $(0,03 \mathrm{mg} / \mathrm{kg})$ e morfina $(0,5 \mathrm{mg} / \mathrm{kg})$ por via intramuscular. Após 15 minutos foi cateterizada a veia cefálica e a induzida a anestesia com propofol $(5 \mathrm{mg} / \mathrm{kg})$ associado ao midazolan $(0,25 \mathrm{mg} / \mathrm{kg})$ pela via intravenosa. Após intubação orotraqueal, a manutenção anestésica foi realizada com isoflurano misturado em oxigênio por meio de um vaporizador universal, empiricamente mantendo a concentração administrada baseada na estabilidade dos parâmetros cardiovasculares.

A determinação da PIA foi realizada logo após a indução anestésica, com o animal em decúbito dorsal e utilizando-se o método de sondagem vesical com sonda uretral de P.V.C calibre $\mathrm{n} \square 8$ para cadelas entre 13 e $25 \mathrm{~kg}$ e calibre $\mathrm{n} \square 10$ para cadelas entre 25 e $37,4 \mathrm{~kg}$. Sob técnica asséptica, foi acoplada à extremidade da sonda uma torneira de três vias, a qual estava conectada a coluna de água de um equipo tipicamente utilizado para aferição da pressão venosa central graduado em $\mathrm{cm} \mathrm{H} 20$. Na outra via da torneira conectou-se um equipo de macro-gotas vinculado a bolsa coletora de urina. Após finalizada a montagem deste sistema, fez-se esvaziamento da bexiga seguida de infusão lenta de 1 $\mathrm{mL} / \mathrm{kg}$ de solução salina a $0,9 \%$ a temperatura ambiente. Passado um minuto abriu-se a torneira de três vias afim de comunicar o equipo de PVC à sonda uretral permitindo que o fluido vesical se encontrasse em equilíbrio com o volume contido na coluna métrica. $\mathrm{O}$ ponto zero do manômetro marcava ao nível da sínfise púbica do animal (Figuras 1 e 2 2). Após a estabilização do menisco da coluna de solução salina, o valor foi registrado baseado na altura da coluna em relação ao ponto zero (Kron, Harman e Nolan, 1984; Lopes, 2010).

Para testar se houve correlação significativa entre o peso dos animais e a PIA, obteve-se o coeficiente de correlação pelo teste paramétrico de Pearson.

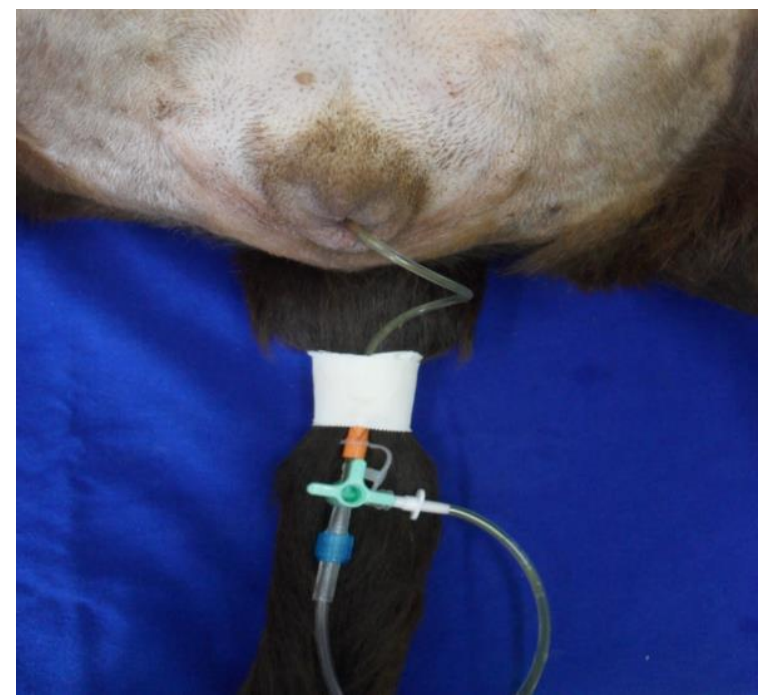

Figura 1 - Cateterização uretral. A sonda uretral de P.V.C. está acoplada a uma torneira de três vias, a qual está conectada a um equipo de macro gotas vinculado a uma bolsa coletora de urina (seta vermelha) e a uma coluna de água a partir de um equipo de PVC (seta amarela). 


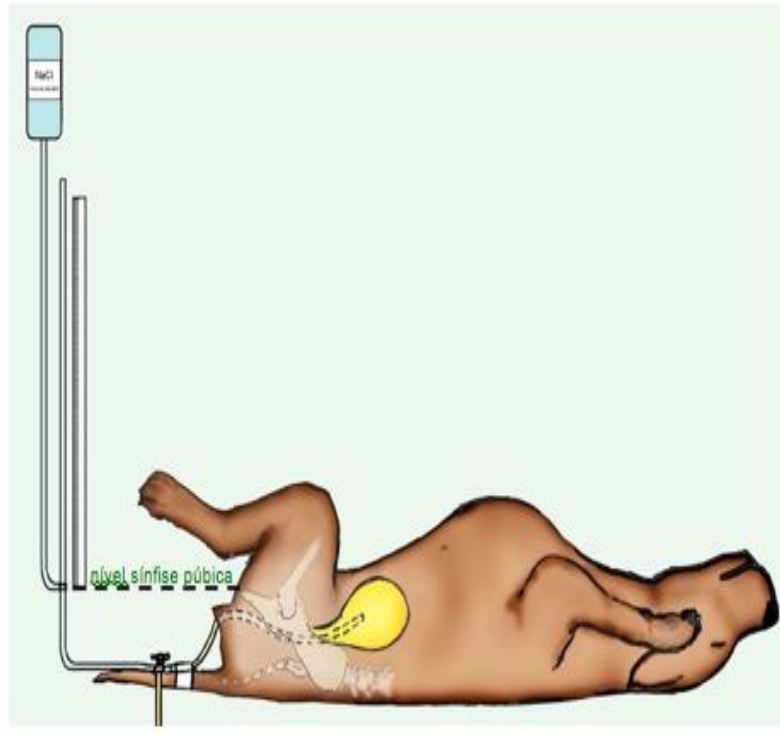

Figura 2 - Visão esquemática do sistema de aferição da pressão intra-abdominal pela método vesical de uma fêmea da espécie canina em decúbito dorsal. Notar o ponto zero como sendo a linha da sínfise púbica.

\section{RESULTADOS}

A média de peso da população de cães do sexo feminino utilizados neste estudo foi de $21,49 \mathrm{~kg}$, com intervalo de 13 a $37,4 \mathrm{~kg}$. O intervalo da pressão intra-abdominal dos 29 animais avaliados foi de 0 à $4,3 \mathrm{cmH} 20$, com média de 1,79 $( \pm 1,39) \quad \mathrm{cmH} 20$ e mediana de $1,9 \mathrm{cmH} 20$. A frequência com que cada valor ocorreu, está apresentando na figura 3 .
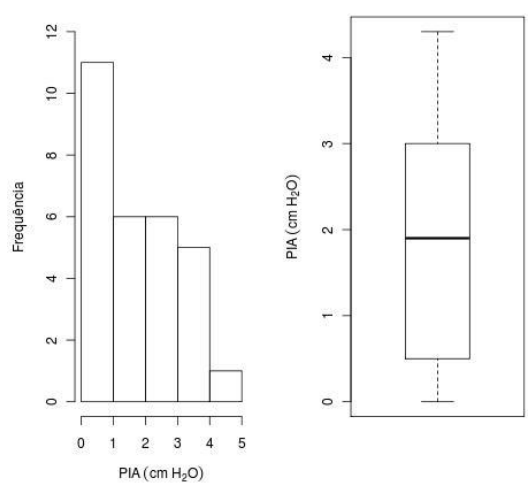

Figura 3 - Distribuição dos valores da PIA em $\mathrm{cmH} 20$ de 29 cães do sexo feminino imediatamente após indução anestésica, pelo método de sondagem uretral com sonda de P.V.C.

Foi observada uma tendência de aumento da PIA, com relação ao aumento do peso dos animais representado no diagrama de dispersão de PIA e PESO com uma reta de Mínimos na figura 4.

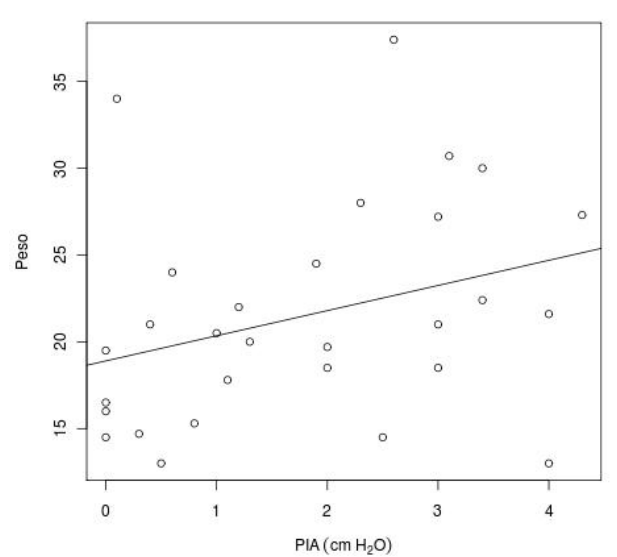

Figura 4 - Diagrama de dispersão PIA e PESO em cadelas submetidas à mensuração da PIA com sonda uretral de P.V.C. logo após indução anestésica.

Tabela 1 - Resultado do coeficiente de correlação de Pearson entre Peso e Pressão Intra-Abdominal em cadelas submetidas à mensuração da PIA com sonda uretral de P.V.C. logo após indução anestésica.

\begin{tabular}{lll} 
Coeficiente & Estatística & p-valor \\
\hline Pearson & $\mathrm{T}=1,75$ & 0,09
\end{tabular}

\section{DISCUSSÃO}

A média da PIA descrita anteriormente por outros grupos de pesquisa para cadelas hígidas de 4,5, 3,51 e $8,7 \mathrm{cmH} 20$ (Conzemius et al., 1995; Lopes, 2010; Way e Monnet, 2014), foi superior aos valores encontrados neste estudo de $1,79 \mathrm{~cm}$ H20 $(1,32 \mathrm{mmHg})$, com intervalo de variação (0 a 4,3 cm H20 / 0 a 3,16 $\mathrm{mmHg}$ ). Estes estudos realizaram a mensuração da PIA pelo mesmo método vesical, porém com a diferença de terem utilizado a sonda de Foley ao 
invés da sonda de P.V.C., além da mensuração ter sido feita em decúbito lateral. A sonda de P.V.C. apresenta parede mais rígida, impedindo a dissipação da pressão ao longo do seu trajeto. Desta maneira os valores tendem ser mais altos e fidedignos. Quanto mais duro for o material da sonda, menores são as chances da parede se expandir com a elevação da pressão em seu interior, dessa forma as pressões serão conduzidas fielmente em seu interior sem comprometer os valores reais da pressão (Bregagnollo et al., 2007). Porém, como os valores pressóricos obtidos são muito baixos, a troca do sonda não pode influir nesta diferença.

Uma das explicações adequadas para a diferença de valores de PIA entre os pesquisadores, pode estar relacionada ao peso e escore corporal dos animais avaliados. Neste estudo foi observado que há uma correlação significativa entre a PIA e o peso, corroborando com vários estudos realizados em pessoas, que comprovaram que a PIA sofre um incremento em indivíduos com índice maior de massa corporal (De Keulenaer et al., 2009a; Mclntosh et al., 2003; Varela, Hinojosa e Nguyen, 2009; Wilson et al., 2010). Lopes (2010) mensurou a PIA em cães com até 78 $\mathrm{Kg}$, encontrando médias maiores, fato que já era esperado.

No presente estudo e nos demais em medicina veterinária, não foram apresentados o escore corporal dos animais, 0 que parece ser mais importante neste contexto do que o próprio peso, pois existe uma grande quantidade de raças e portes e, consequentemente, extensa variedade de peso. Mais precisamente, há uma correlação direta do diâmetro abdominal com valores maiores de PIA em seres humanos (Lambert, Marceau e Forse, 2005). Pacientes com obesidade mórbida e maiores valores de PIA (12
$\mathrm{mmHg}$ ), apresentam ainda mais comorbidades, como doença de refluxo gastroesofágico, hérnia abdominal, diabetes, hipertensão arterial sistêmica e insuficiência venosa.

Mudanças na posição do corpo (ou seja, decúbito lateral, dorsal, cabeça mais elevada do restante do corpo) também tem impacto sobre a precisão das aferições da PIA (De Waele et al., 2009). Indica-se a posição supina para pessoas, (proporcional ao decúbito dorsal para os quadrúpedes) com 0 corpo no mesmo nível, sem qualquer angulação. Mensurações realizadas em posição supina e lateral, mostraram que quando mensurada em decúbito lateral os valores da PIA são significativamente mais altos, o mesmo ocorre em situações nas quais a cabeça está 15 , 30 ou 45 graus acima do restante do corpo (Cheatham et al., 2009; De Keulenaer et al., 2009a; De Keulenaer et al., 2009b). Diante disso e da ausência de estudos em cães sobre o efeito da posição na determinação da PIA, optou-se por realizar as mensurações em decúbito dorsal, apesar de todos os outros estudos realizados em medicina veterinária mensuraram a PIA em decúbito lateral. Um único estudo realizado em felinos não encontrou diferenças significativas entre as posições lateral e dorsal (Lopes, 2010).

Após inúmeros estudos médicos, atualmente, o consenso de especialistas da WSACS preconiza instilar o volume de $25 \mathrm{~mL}$ de solução fisiológica na bexiga (Malbrain et al., 2006). Esta recomendação de volume fixo não é adequada em medicina veterinária, pois o tamanho de bexiga é muito variável, especialmente nos cães, devido variedade de raças e portes. Infusão de volume em excesso pode acarretar em superestimação da PIA, devido a hiperdistensão da bexiga, podendo levar a contração muscular por deflagrar 0 reflexo de micção (Kimball et al., 2009). 
Quando foi comparada a mensuração da PIA em crianças pelos métodos indiretos transvesical instilando 1 , 3 e $5 \mathrm{~mL} / \mathrm{kg}$ de solução fisiológica na bexiga e manometria intragástrica por intermédio de uma sonda nasogástrica com o método direto com catéter de diálise peritoneal, concluiu-se que 0 método indireto mais fidedigno é o transuretral com a instilação de $1 \mathrm{~mL} / \mathrm{Kg}$ de solução fisiológica na bexiga (Davis et al., 2005). Um estudo em felinos mensurou a PIA com a administração de $0,1 \mathrm{~mL}, 0,5$ e $1 \mathrm{~mL}$ sem detectar diferença estatística nos resultados (Lopes, 2010). Way e Monnet (2014), compararam os valores da PIA aferidos pelo método indireto $(0,25 \mathrm{~mL}, 0,5 \mathrm{~mL}$, $1 \mathrm{~mL}$ e $2 \mathrm{~mL} / \mathrm{kg}$ ) com o método direto, concluiundo que o volume ideal é o de 1 $\mathrm{mL} / \mathrm{kg}$. Os demais estudos em cães também utilizaram o volume de $1 \mathrm{~mL} / \mathrm{kg}$ de solução fisiológica (Drellich, 2000; Lopes, 2010). No presente estudo, optou-se por este volume, a fim de evitar sub ou superstimação da PIA e adequar o volume de acordo com $\mathrm{O}$ porte do animal.

Relatou-se um caso de SCA em um cão com babesiose, no qual a mensuração da PIA se deu com a instilação de $50 \mathrm{~mL}$ de solução fisiológica em um cão com $30 \mathrm{Kg}$ (Joubert et al., 2007). A não padronização dos valores de enchimento vesical pode contribuir para a detecção de valores mais altos de PIA.

No entanto, mesmo que estes resultados do presente estudo em relação a correlação da PIA e peso estejam em conformidade com os dados da literatura, a variedade de portes entre os cães é grande e possivelmente o tamanho da bexiga não acompanhe proporcionalmente essa variação, descartando a possibilidade de que 0 volume de $1 \mathrm{~mL} / \mathrm{Kg}$ seja ideal em todos os portes de cães, sem acarretar superestimação da PIA, devido a hiperdistensão da bexiga.

\section{CONCLUSÃO}

A média da PIA de cadelas anestesiadas com propofol e isoflurano pelo método proposto foi de $1,78 \mathrm{cmH} 20$ $( \pm 1,39)$ e a mediana de $1,9 \mathrm{cmH} 20$. Os valores da PIA tem relação direta com o peso dos animais.

\section{NOTAS INFORMATIVAS}

Reservado ao parecer CEUA.

\section{REFERÊNCIAS}

ANDRADE, I.J. A síndrome de compartimento do abdome. Revista de Medicina Ribeirão Preto, v. 31, p. 563-7, 1998.

BALL, C.G.; KIRKPATRICK, A.W.; McBETH, P. The secondary abdominal compartment syndrome: not just another post-traumatic complication. Canadian Journal of Surgery, v. 51 , n. 5, p. 399405, 2008.

BREGAGNOLLO, et al. Aspectos metodológicos relacionados aos sistemas manométricos utilizados em estudos hemodinâmicos. Revista Brasileira de Cardiologia Invasiva, v. 15, n. 4, p. 421-431, 2007.

CASTELHANOS, G. PIÑERO, A. FERNÁNDEZ, J. La hipertensión intraabdominal y el síndrome compartimental abdominal: que debe saber y cómo debe tratarlos el cirujano? Cirugía Española, v. 81, n. 1, p. 4-11, 2007.

CHEATHAM, M.L. et al. Critical Care Medicine, v. 37, n. 7, p. 2187-90, 2009.

CHEN, $\mathrm{H}$. et al. Abdominal compartment syndrome in patients with severe acute pancreatitis in early stage. World Journal 
of Gastroenterology, n. 14, v. 22, p. 3541-3548, 2008.

COHEN, R.V. et al. Alterações sistêmicas e metabólicas da cirurgia laparascópica. Revista Brasileira de Videocirurgia, v.1, n.2, 2003.

CONZEMIUS, M.G. et al. Clinical determination of preoperative and postoperative intra-abdominal pressures in dogs. Veterinary Surgery, v. 24, p. 195-201, 1995.

DAVIS, P.J. et al. Comparison of indirect methods of measuring intra-abdominal pressure in children. Intensive Care Medicine, v. 31, n. 3, p. 210-5, 2009.

DE KEULENAER, B.L. et al. Intraabdominal pressure measurements in lateral decubitus versus supine position. Acta Clinica Belgica, v. 64, n. 3, p. 96976, 2009a.

DE KEULENAER, B.L. et al. What is normal intra-abdominal pressure and how is it affected by positioning, body mass and positive end-expiratory pressure? Intensive Care Medicine, v. 35, n. 6, p. 969-76, 2009b.

De WAELE, J.J., et al. Recomendations for research from the Internacional Conference of Experts on Intraabdominal Hypertension and Abdominal Compartment Syndrome. Acta Clinica Bélgica, v. 64, n. 3, p. 203-209, 2009.

DRELLICH, S. Intra-abdominal pressure and abdominal compartment syndrome. Compedium on Continuing Education fot the Practicing Veterinarian, v. 22, n. 8, p. 764-768, 2000.

FETNER, M.; PRITTIE, J. Evaluation of transvesical intra-abdominal pressure measurement in hospitalized dogs. Journal of Veterinary Emergency and Critical Care, v. 22, n. 2, p. 230-238, 2012.

JOUBERT, K.E., et al. Abdominal compartment syndrome in a dog with babesiosis. Journal of Veterinary Emergency of Veterinary and Critical, v. 17, n. 2, p.184-190, 2007.

KIMBALL, E.J. et al. A comparison of infusion volumes in the measurement of intra-abdominal pressure. Journal of Intensive Care Medicine, v. 24, n. 4, p. 261-8, 2009.

KRON, I.L; HARMAN, P.K.; NOLAN, S.P. The measurement of intraabdominal pressure as a criterion for abdominal re-exploration. Annals of Surgery, v. 199, n. 1, p. 28-30, 1984.

LAMBERT, D.M.; MARCEAU, S.; FORSE, R.A. Intra-abdominal pressure in the mordidly obese. Obesity Surgery, v. 15, n. 9, p. 1225-32, 2005.

LOPES, A.M.C.M. Fisiopatologia da pressão intra-abdominal em animais de companhia. 59 f. Dissertação (Mestradro Integrado em Medicina Veterinária) Setor de Medicina Veterinária Universidade Técnica de Lisboa, Lisboa, 2010.

MALBRAIN, M.L.N.G.; JONES, F. Intraabdominal pressure measurement techniques. In: IVATURY, $R$. et al. Abdominal Compartment Syndrome. Georgetown: Landes Bioscience, p. 1968, 2006.

MALBRAIN, M.L.N.G. et al. Results from the International Conference of Experts on Intra-abdominal Hypertension and Abdominal Compartment Syndrome. I. Definitions. Intensive Care Medicine, v. 32, n. 11, p. 1722-1732, 2006.

MCINTOSH, S. et al. Relationship of abdominal pressure and body mass index in men with LUTS. Neurourol Urodyn, v. 22, n. 6, p. 602-5, 2003.

ONICHIMOWSKI, D. et al. Measurement of intra-abdominal pressure in clinical practice. Anaesthesiology Intensive Therapy, v. 42, n. 2, p. 96-101, 2010.

PRADO, L.F.A. et al. Pressão intraabdominal em pacientes com trauma 
abdominal. Revista do Colégio Brasileiro de Cirurgiões, v. 32, n. 2, p. 83-89, 2005.

OTTO, J. et al. Clinical evaluation of an air-capsule technique for the direct measurament of intra-abdominal pressure after elective abdominal surgery. MC Surgery, v. 8, n. 18, 2008.

VARELA, J.E; HINOJONOSA, M. NGUYEN, N. Correlations between intra-abdominal pressure and obesityrelated co-morbidities. Surgery for Obesity and Related Diseases, v. 5, n. 5, p. 524-528, 2009.

WILSON, A. et al. Intra-abdominal pressure and the morbidly obese patients: the effect of body mass index. Journal of Trauma-Injury Infection \& Critical Care, v. 69, n. 1, p. 78-83, 2010.

WAY, L.I.; MONNET, E. Determination and validation of volume to be instilled for standardized intra-abdominal pressure measurement in dogs. Journal of Veterinary Emergency and Critical Care, v. 24, n. 4, p. 403-407, 2014. 